\title{
Unconventional metamagnetic electron states in orbital band systems
}

\author{
Wei-Cheng Lee ${ }^{1}$ and Congjun $\mathrm{Wu}^{1}$ \\ ${ }^{1}$ Department of Physics, University of California, San Diego, CA 92093
}

\begin{abstract}
We extend the study of the Fermi surface instability of the Pomeranchuk type into systems with orbital band structures, which are common features in transition metal oxides. Band hybridization significantly shifts the spectra weight of the Landau interactions from the conventional $s$-wave channel to unconventional non- $s$-wave channels, which results in anisotropic (nematic) Fermi surface distortions even with ordinary interactions in solids. The Ginzburg-Landau free energy is constructed by coupling the charge-nematic, spin-nematic and ferromagnetic order parameters together, which shows that nematic electron states can be induced by metamagnetism. The connection between this mechanism to the anisotropic metamagnetc states observed in $\mathrm{Sr}_{3} \mathrm{Ru}_{2} \mathrm{O}_{7}$ at high magnetic fields is studied in a multi-band Hubbard model with the hybridized quasi-one dimensional $d_{x z}$ and $d_{y z}$-bands.
\end{abstract}

PACS numbers: 71.10.Ay, 75.10.-b,75.30Kz

\section{INTRODUCTION}

Pomeranchuk instabilities are a large class of Fermi surface instabilities in both density and spin channels, each of which can be further decomposed into different partial wave channels $\stackrel{1}{*}$. This class of instabilities in the non-s-wave density channel result in uniform but anisotropic (nematic) electron liquid states ${ }^{2}$, which have recently attracted a great deal of attention in recent

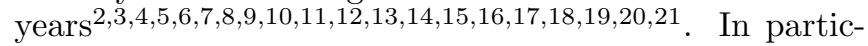
ular, these instabilities have been studied in the context of doped Mott insulators ${ }^{22}$, high $\mathrm{T}_{c}$ materials ${ }^{3,22}$, and quantum Hall systems in nearly half-filled Landau levels ${ }^{23,24}$. Experimental evidence has also been found in ultra-high mobility two-dimensional electron gases and quantum wells in large magnetic fields 25,26,27.

Non-s-wave spin channel Pomeranchuk instabilities are "unconventional magnetism" in analogy to unconventional superconductivity, which have been extensively investigated $2,4,5,28,29,30,31,32$. In Refs ${ }^{28}$ by $\mathrm{Wu}$ and Zhang, these states are classified as isotropic and anisotropic phases dubbed $\beta$ and $\alpha$-phases as the counterparts of ${ }^{3} \mathrm{He}-B$ (isotropic) and $A$ (anisotropic) phases, respectively. The $\beta$-phases have circular or spherical Fermi surfaces with topologically non-trivial spin configurations in momentum space $\frac{28}{2}$. In the $\beta$-phase, the relative spin-orbit symmetry is broken, a concept introduced in the context of ${ }^{3} \mathrm{He}-\mathrm{B}$ phase, while essentially the overall rotational symmetry is not. The $\alpha$-phases are anisotropic electron liquid crystal states with spin degree of freedom, which have been studied by many groups: the $p$-wave phase was first studied by Hirsch 31,32 under the name of the "spin-split" state, and was also proposed by Varma et al. $\underline{50}$. phenomenon in the heavy fermion compound $\mathrm{URu}_{2} \mathrm{Si}_{2}$; the $d$-wave phase was studied by Oganesyan et al. . $^{2}$ under the name of "nematic-spin-nematic" phase. Systematic studies of the ground state properties and collective excitations in both the anisotropic $\alpha$ and isotropic $\beta$-phases has been performed by $\mathrm{Wu}$ et al. $\stackrel{28,29}{ }$. Very recently,
Chubukov and Maslov found that when approaching the ferromagnetic quantum critical point, the $p$-wave channel spin Pomeranchuk instability develops prior to the developing of ferromagnetic instability ${ }^{33}$.

Although unconventional magnetism has not been convincingly identified in experiments, a spontaneous nematic electron liquid has been observed in the ultra-clean samples of the bilayer ruthenate $\mathrm{Sr}_{3} \mathrm{Ru}_{2} \mathrm{O}_{7} 734,35,36$, which has arouse much research interest $\frac{4,37,38,39,40,41,42,43}{}$. $\mathrm{Sr}_{3} \mathrm{Ru}_{2} \mathrm{O}_{7}$ is a metallic itinerant system with the tetragonal $\mathrm{RuO}_{2}(a b)$ planes. It is paramagnetic at zero magnetic field, and develops two consecutive metamagnetic transitions in the external magnetic field $B$ perpendicular to the $a b$-plane at 7.8 and 8.1 Tesla below $1 \mathrm{~K}$, respectively. In the state between two metamagnetic transitions, the resistivity measurements show a strong spontaneous in-plane anisotropy (nematic) along the $a$ and $b$ axis with no noticeable lattice distortions, thus this effect is of electronic origin. It can be interpreted as a nematic state with the anisotropic distortion of the Fermi surface of the majority spin polarized by the external magnetic field in Ref ${ }^{35}$, which is essentially a mixture of the $d$ wave Pomeranchuk instabilities in both density and spin channels.

In spite of years of intensive research, most theories remain phenomenological without considering the actual orbital band structures of $\mathrm{Sr}_{3} \mathrm{Ru}_{2} \mathrm{O}_{7} 37,38,39$. Two key questions have not been answered satisfactorily after years of intensive research. First, $\mathrm{Sr}_{2} \mathrm{Ru}_{3} \mathrm{O}_{7}$ is a $t_{2 g}$-band system, having both quasi-one dimensional bands of $d_{x z}$ and $d_{y z}$ and the two-dimensional bands of $d_{x y}$. Which are responsible for the nematic behavior? Second, the nematic states require strong exchange interactions in the $d$-wave channel, but the usual exchange interaction from Coulomb repulsion is mostly in the $s$-wave channel. Microscopic theories based on the single band picture of $d_{x y}$ combined with the van Hove singularity have been nicely developed ${ }^{4,42,43}$. However, their models need a large $d$ wave channel exchange interaction which was introduced by hand. It is difficult to justify the microscopic origin of this interaction in terms of Coulomb interaction. Fur- 
thermore, these theories do not address the fact that the nematic ordering does not appear in the single layer compound $\mathrm{Sr}_{2} \mathrm{RuO}_{4}$, which has a similar band structure of $d_{x y}$.

In this article, we provide a natural answer to these two key questions by extending the theory of Pomeranchuk instabilities into multi-orbital systems. We point out that it is the hybridized quasi-one-dimensional $d_{x z}$ and $d_{y z}$ bands instead of the $d_{x y}$ band that are responsible for the nematic ordering based on the following reasoning. The key difference of electronic structures between $\mathrm{Sr}_{3} \mathrm{Ru}_{2} \mathrm{O}_{7}$ and $\mathrm{Sr}_{2} \mathrm{RuO}_{4}$ is the bilayer splitting, which is prominent for the quasi-one dimensional bands of $d_{x z}$ and $d_{y z}$ but small for the two-dimensional bands of $d_{x y}$. It is natural to expect that the spontaneous nematic behavior occurs in the bands of $d_{x z}$ and $d_{y z}$ and is accompanied by an orbital ordering. Furthermore, the orbital band hybridization between them shifts a significant spectra weight of the exchange interaction into the $d$-wave channel, thus the nematic ordering can arise from the conventional multi-band Hubbard interactions. This mechanism also applies to other strongly correlated orbital systems.

This paper is organized as follows. We first present a heuristic picture to illustrate the idea how orbital hybridization enhances the Landau interaction in non- $s$ wave channels in Sect. III. In Sect. III, a phenomenological Ginzburg-Landau free energy is constructed to explain the two consecutive metamagnetic transitions and the nematic phase in between. In Sect. IV, we use a microscopic multi-orbital Hubbard model based on the quasi-one dimensional $d_{x z}$ and $d_{y z}$-bands to explain the appearance of the nematic state. Conclusions and outlooks are made in Sect. V.

\section{LANDAU INTERACTIONS MODIFIED BY ORBITAL HYBRIDIZATION}

In this section, we present a heuristic picture to illustrate the enhancement of the non-s-wave Landau interactions from orbital band hybridizations. For a single band system without orbital structures, the Landau interaction functions can be simply expressed at the HartreeFock level in the density and spin channels as:

$$
\begin{aligned}
& f^{s}\left(\vec{p}_{1}, \vec{p}_{2}\right)=V(\vec{q}=0)-\frac{1}{2} V\left(\left|\vec{p}_{1}-\vec{p}_{2}\right|\right), \\
& f^{a}\left(\vec{p}_{1}, \vec{p}_{2}\right)=-\frac{1}{2} V\left(\left|\vec{p}_{1}-\vec{p}_{2}\right|\right),
\end{aligned}
$$

where $V(\vec{p})$ is the Fourier transform of the two-body interaction $V\left(\left|\vec{r}_{1}-\vec{r}_{2}\right|\right)$, say, the Coulomb interaction. The high partial wave channel components of $V$ are usually weak, thus the condition for Pomeranchuk instabilities in high partial channels is more stringent than that of the $s$-wave instability of ferromagnetism.

This situation is significantly changed in multi-band systems with non-trivial orbital hybridization. Let us consider a simplified two dimensional example of the hybridized bands between $d_{x z}$ and $d_{y z}$ and assume the single particle eigenstates around the Fermi surface takes the form of

$$
\begin{aligned}
\left|\psi_{\sigma}(\vec{p})\right\rangle & =e^{i \vec{p} \cdot \vec{r}}|u(\vec{p})\rangle \otimes \chi_{\sigma}, \\
|u(\vec{p})\rangle & =\cos \phi_{p}\left|d_{x z}\right\rangle+\sin \phi_{p}\left|d_{y z}\right\rangle
\end{aligned}
$$

where $\phi_{p}$ is the azimuthal angle of $\vec{p} ;|u(\vec{p})\rangle$ is the Bloch wave function with the internal orbital configurations; $\chi_{\sigma}(\sigma=\uparrow, \downarrow)$ are the spin eigenstates. This orbital structure has no effects on the Hartree interaction between two electrons with opposite spins, while it significantly changes the Fock exchange interaction of two electrons of the same spin which are not completely indistinguishable any more. Consequentially, the exchange interaction between them acquires an extra form factor describing the inner product of their orbital configurations as

$$
-V\left(\vec{p}_{1}-\vec{p}_{2}\right)\left|\left\langle u\left(p_{1}\right) \mid u\left(p_{2}\right)\right\rangle\right|^{2} .
$$

The Landau interaction functions change to

$$
\begin{aligned}
f^{s}\left(\vec{p}_{1}, \vec{p}_{2}\right) & =V(\vec{q}=0)-\frac{1}{4}\left[1+\cos 2\left(\phi_{p_{1}}-\phi_{p_{2}}\right)\right] \\
& \times V\left(\vec{p}_{1}-\vec{p}_{2}\right), \\
f^{a}\left(\vec{p}_{1}, \vec{p}_{2}\right) & =-\frac{1}{4}\left[1+\cos 2\left(\phi_{p_{1}}-\phi_{p_{2}}\right)\right] V\left(\vec{p}_{1}-\vec{p}_{2}\right) .
\end{aligned}
$$

Therefore even if the bare interaction $V\left(\vec{p}_{1}-\vec{p}_{2}\right)$ is dominated by the $s$-wave channel, the extra $d$-wave factor arising from the orbital hybridization shifts a significant weight into the $d$-wave channel.

\section{THE GINZBERG-LANDAU THEORY}

The Eq. 4 above implies that a strong ferromagnetic or metamagnetic ( $s$-wave) tendency also enhances the nematic ordering ( $d$-wave). Before constructing the microscopic theory, we illustrate this point through a Ginzburg-Landau free energy formalism which includes the coupling among the ferromagnetic order $m$, the charge nematic order $n_{c}$, and spin nematic order $n_{s p}$.

In the square lattice, the two different nematic channels $d_{x^{2}-y^{2}}$ and $d_{x y}$ belong to non-equivalent representations. Only the $d_{x^{2}-y^{2}}$ channel instability is experimentally observed and thus is kept. Due to the experimentally observed anisotropy between the $z$-axis and the $a b$-plane, we only keep the $z$-component of spin and spinnematic orders. The Ginzburg-Landau free energy is constructed as

$$
\begin{aligned}
F(h) & =F(m)-h m+r_{c} n_{c}^{2}+r_{s p} n_{s p}^{2}+g_{c} n_{c}^{4}+g_{s} n_{s p}^{4} \\
& +g(m) n_{c} n_{s p}
\end{aligned}
$$

where $F(m)$ is the magnetic order contribution to the free energy as an even function of $m ; h$ is the external magnetic field; $r_{c} \propto\left(2+F_{2}^{s}\right)$ and $r_{s p} \propto\left(2+F_{2}^{a}\right)$ are the 

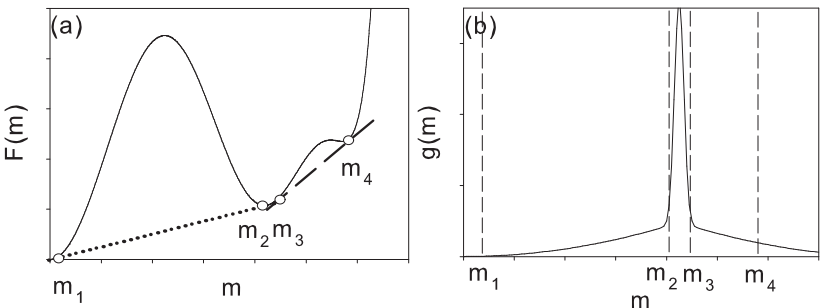

FIG. 1: a) The sketch of the magnetic part $F(m)$ in the Ginzburg-Landau free energy. The slopes of two common tangent lines mark the magnetic fields of meta-magnetic transitions. b) The dimensionless coupling function $g^{\prime}(m)$ has a peak distribution between two consecutive matamagnetic transitions.

mass terms of charge and spin nematic orders, respectively; $n_{c, s p}$ are defined as:

$$
\begin{aligned}
n_{c} & =\sum_{\vec{k}, \sigma}\left\langle\psi_{\sigma}^{\dagger}(\vec{k}) \psi_{\sigma}(\vec{k})\right\rangle f(\vec{k}), \\
n_{s p} & =\sum_{\vec{k}, \sigma}\left\langle\psi_{\alpha}^{\dagger}(\vec{k}) \sigma_{z, \alpha \beta} \psi_{\beta}(\vec{k})\right\rangle f(\vec{k}),
\end{aligned}
$$

where $f(\vec{k})$ is a form factor exhibiting the $d_{x^{2}-y^{2}}$ symmetry; $g(m)$ is the coupling function between $n_{c}$ and $n_{s p}$ which is an odd function of $m$ as required by time-reversal symmetry. The experimentally observed two consecutive metamagnetic transitions can be reproduced by a suitable designed form of $F(m)$ sketched in Fig. 1 $a$ where only the part with $m>0$ is shown. It has two common tangent lines marked with dotted and dashed lines, which touch the curve at points of $m_{1,2}$ and $m_{3,4}$, respectively. In the external magnetic field $h$, the solution of $m$ satisfies the equation:

$$
\frac{d}{d m} F(m)=h
$$

Therefore, the slopes of the two common tangent lines $h_{1,2}$ can be interpreted as the fields at which magnetization jumps from $m_{1}$ to $m_{2}$ and from $m_{3}$ to $m_{4}$, i.e., metamagnetic transitions occur. When $h$ lies between these two transitions, the magnetization $m$ evolves continuously.

The development of nematic orders between the two successive metamagnetic transitions is triggered by the $g(m)$-term. Although the nematic instability is enhanced by Eq. 4, they are still a weaker instability compared to ferromagnetism (metamagnetism). This is because the condition for Pomeranchuk instability in the $d$-wave channel in $2 \mathrm{D}$, i.e., $F_{2}^{s, a}<-2$, is more stringent that in the $s$-wave channel, i.e. $F_{0}^{a}<-1$. We assume that the charge and spin nematic channels are close to be critical but not yet, i.e., $r_{c, s p}$ are small but positive. Due to the hybridization term of $g(m)$, the eigen-order parameters

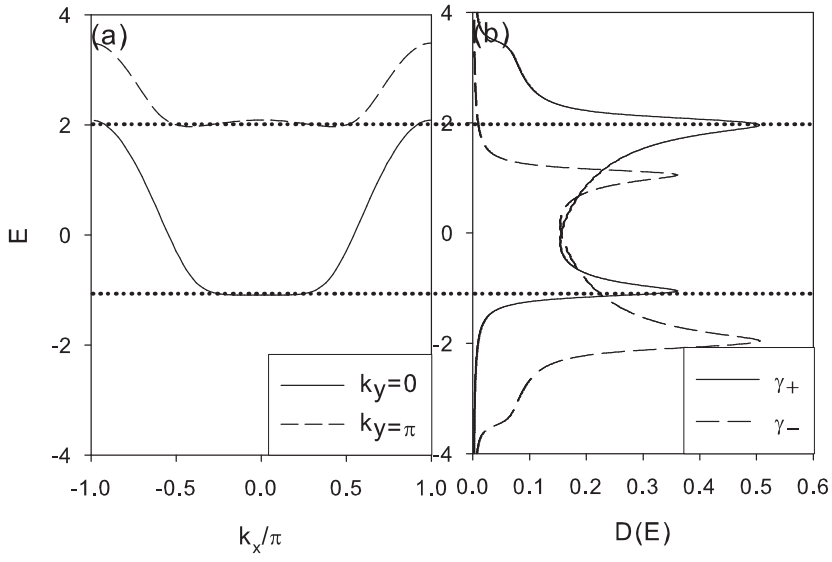

FIG. 2: (a) Dispersion of the $\gamma_{+}$band for $\vec{k}=\left(k_{x}, 0\right)$ and $\left(k_{x}, \pi\right)$. (b) The DOS of the $\gamma_{ \pm}$bands whose peaks corresponds to the van Hove singularities at $\vec{k}=(0,0),(0, \pi),(\pi, 0)$.

arise from the diagonalization of the quadratic terms of $n_{c, s p}$ as:

$$
\begin{aligned}
& n_{+}=\cos \theta n_{c}+\sin \theta n_{s}, \\
& n_{-}=-\sin \theta n_{c}+\cos \theta n_{s},
\end{aligned}
$$

where $\tanh 2 \theta=2 g(m) /\left(r_{c}-r_{s p}\right)$. The corresponding eigenvalues read:

$$
r_{ \pm}=\frac{1}{2}\left\{r_{c}+r_{s p} \pm \sqrt{\left(r_{c}-r_{s p}\right)^{2}+4[g(m)]^{2}}\right\} .
$$

The critical coupling for the $n_{+}$channel to develop the instability is

$$
\left[g^{\prime}(m)\right]^{2} \equiv \frac{[g(m)]^{2}}{4 r_{c} r_{s p}}>1
$$

In the presence of the van Hove singularity of DOS, which is a common mechanism for meta-magnetism, all the parameters in the GL free energy could change significantly so that the distribution of the dimensionless coupling function $g^{\prime}(m)$ may not be smooth. For the nematic order only occuring in the regime between two metamagnetic transitions, $g^{\prime}(m)$ must have a peak satisfying Eq. 10 at $m_{2}<m<m_{3}$ but is below the critical value elsewhere as sketched in Fig. 1 $b$. Roughly speaking, the underlying physics is that metamagnetism pushes the majority Fermi surface even closely to the van Hove singularity which finally drives the nematic ordering ${ }^{4}$. The minority Fermi surfaces is pushed away from critical. In the following we will confirm this mechanism explicitly in a microscopic calculation.

\section{MICROSCOPIC THEORY FOR THE BILAYER $\mathrm{Sr}_{3} \mathrm{Ru}_{2} \mathrm{O}_{7}$}

We next make the connection to $\mathrm{Sr}_{3} \mathrm{Ru}_{2} \mathrm{O}_{7}$ by exploiting a microscopic model with $d_{x z}$ and $d_{y z}$-orbital bands. 
They have the bonding and anti-bonding bands with a large bilayer splitting at the order of their band widths. Only the bonding bands, the even combination of the bilayer orbitals, are considered because their Fermi surfaces are close to van Hove singularity which enhances interaction effects. Because of orbital hybridization, they form closed Fermi surfaces. As moving around this Fermi surface, the orbital configuration varies between $d_{x z}$ and $d_{y z}$ exhibiting a $d$-wave pattern, thus the mechanism illustrated in Sect. II] applies.

The band Hamiltonian $H_{0}$ reads

$$
\begin{aligned}
H_{0} & =\sum_{\vec{k} \sigma}\left\{\epsilon_{x z, \vec{k}} d_{x z \vec{k} \sigma}^{\dagger} d_{x z, \vec{k} \sigma}+\epsilon_{y z, \vec{k}} d_{y z, \vec{k} \sigma}^{\dagger} d_{y z, \vec{k} \sigma}\right. \\
& \left.+\lambda_{\vec{k}}\left(d_{x z, \vec{k} \sigma}^{\dagger} d_{y z, \vec{k} \sigma}+\text { h.c. }\right)\right\}
\end{aligned}
$$

with

$$
\begin{aligned}
\epsilon_{x z, \vec{k}} & =-2 t_{\|} \cos k_{x}-2 t_{\perp} \cos k_{y}-4 t^{\prime} \cos k_{x} \cos k_{y} \\
\epsilon_{y z, \vec{k}} & =-2 t_{\perp} \cos k_{x}-2 t_{\|} \cos k_{y}-4 t^{\prime} \cos k_{x} \cos k_{y} \\
\lambda_{\vec{k}} & =4 t^{\prime \prime} \cos k_{x} \cos k_{y} .
\end{aligned}
$$

$t_{\|}$and $t_{\perp}$ are the nearest neighbor longitudinal and transverse hopping integrals for the $d_{x z}$ and $d_{y z}$-orbitals; $t^{\prime}$ and $t^{\prime \prime}$ are the next-nearest neighbor intra and interorbital hopping, respectively. The resulting diagonalized band Hamiltonian is:

$$
H_{0}=\sum_{\vec{k} \sigma} E_{\vec{k}}^{+} \gamma_{+\vec{k} \sigma}^{\dagger} \gamma_{+\vec{k} \sigma}+E_{\vec{k}}^{-} \gamma_{-\vec{k} \sigma}^{\dagger} \gamma_{-\vec{k} \sigma}
$$

where

$$
E_{\vec{k}}^{ \pm}=\frac{1}{2}\left[\epsilon_{x z, \vec{k}}+\epsilon_{y z, \vec{k}} \pm \sqrt{\left(\epsilon_{x z, \vec{k}}-\epsilon_{y z, \vec{k}}\right)^{2}+4 h_{\vec{k}}^{2}}\right](14)
$$

and the band eigen-operators reads

$$
\begin{aligned}
& \gamma_{+, \vec{k} \sigma}=\cos \phi_{\vec{k}} d_{x z, \vec{k} \sigma}+\sin \phi_{\vec{k}} d_{y z, \vec{k} \sigma}, \\
& \gamma_{-, \vec{k} \sigma}=-\sin \phi_{\vec{k}} d_{x z, \vec{k} \sigma}+\cos \phi_{\vec{k}} d_{y z, \vec{k} \sigma}
\end{aligned}
$$

with the hybridization angle $\phi_{\vec{k}}$ satisfying

$$
\tan 2 \phi_{\vec{k}}=\frac{2 \lambda_{\vec{k}}}{\epsilon_{x z, \vec{k}}-\epsilon_{y z, \vec{k}}}=\frac{-4 t^{\prime \prime}}{t_{\|}-t_{\perp}} \frac{\cos k_{x} \cos k_{y}}{\cos k_{x}-\cos k_{y}}
$$

This band hybridization pattern is a lattice version of Eq. 2 with only the $d_{x^{2}-y^{2}}$ channel but not the $d_{x y}$ channel. We choose the parameter values of $\left(t_{\|}, t_{\perp}, t^{\prime}, t^{\prime \prime}\right)=$ $(1.0,0.145,0.0,0.3)$, and plot the density of states (DOS) in Fig 2. Two peaks in the DOS exist in both $\gamma_{ \pm}$ bands, which correspond to the van Hove singularities at $\vec{k}=(0,0),(0, \pi),(\pi, 0)$.

We take the general multi-band Hubbard model that are widely used in literatures for the interactions as

$$
H_{i n t}=U \sum_{i, a=x z, y z} n_{a \uparrow}(i) n_{a \downarrow}(i)+V \sum_{i} n_{x z}(i) n_{y z}(i)
$$

$$
\begin{aligned}
& -J \sum_{i}\left\{\vec{S}_{x z}(i) \cdot \vec{S}_{y z}(i)-\frac{1}{4} n_{x z}(i) n_{y z}(i)\right\} \\
& +\Delta \sum_{i}\left\{d_{x z, \uparrow}^{\dagger}(i) d_{x z, \downarrow}^{\dagger}(i) d_{y z, \downarrow}(i) d_{y z, \uparrow}(i)+h . c .\right\}
\end{aligned}
$$

where $n_{a, \sigma}$ are particle number operators in orbital $a$ with spin $\sigma ; n_{a}=n_{a, \uparrow}+n_{a, \downarrow} ; \vec{S}_{a}$ are spin operators in orbital $a$. The $U$-term is the intra-orbital repulsion; the $V$-term is the inter-orbital repulsion for the spin triplet configuration of two electrons; the $J$-term represents the Hund's rule physics; the $\Delta$-term describes the inter-orbital pairing hopping.

In the absence of the orbital hybridization, it is not conclusive that the Hubbard model can give rise to nematic transitions because on-site interactions only contribute to the $s$-wave channel. We define the charge nematic order $2 n_{c}=n_{x z}-n_{y z}$, the spin nematic order $n_{s p}=S_{x z}^{z}-S_{y z}^{z}$, and the ferromagnetic order $m=$ $S_{x z}^{z}+S_{y z}^{z}$. The mean-field theory in the eigen-basis of $\gamma_{ \pm}$ reads:

$$
H_{m f}=\sum_{\vec{k} \sigma, \alpha= \pm} \xi_{\alpha \sigma} \gamma_{\alpha \vec{k} \sigma}^{\dagger} \gamma_{\alpha \vec{k} \sigma}+V_{m} m^{2}+V_{s p} n_{s p}^{2}+V_{c} n_{c}^{2}
$$

where

$$
V_{m}=\frac{U}{2}+\frac{J}{4}, V_{s p}=\frac{U}{2}-\frac{J}{4}, V_{c}=V+\frac{J}{4}-\frac{U}{2},
$$

and the less important inter-band coupling terms $\gamma_{+\vec{k} \sigma}^{\dagger} \gamma_{-\vec{k} \sigma}$ are dropped. The renormalized single particle spectra become:

$$
\xi_{\alpha \sigma}=E_{\vec{k}}^{\alpha}-\mu-\sigma V_{m} m-\alpha\left(V_{c} n_{c}+\sigma V_{s p} n_{s p}\right) \cos 2 \phi_{k},
$$

where $\phi_{k}$ are the hybridization angle defined in Eq. 16 . The self-consistent equations for the order parameters read:

$$
\begin{aligned}
n_{c} & =\frac{1}{2 N} \sum_{k, \sigma}\left\{n_{f,+, \sigma}(\vec{k})-n_{f,-, \sigma}(\vec{k})\right\} \cos 2 \phi_{\vec{k}} \\
n_{s p} & =\frac{1}{2 N} \sum_{\vec{k}, \sigma} \sigma\left\{n_{f,+, \sigma}(\vec{k})-n_{f,-, \sigma}(\vec{k})\right\} \cos 2 \phi_{\vec{k}} \\
m & =\frac{1}{2 N} \sum_{\vec{k}, \sigma} \sigma\left\{n_{f,+, \sigma}(\vec{k})+n_{f,-, \sigma}(\vec{k})\right\}
\end{aligned}
$$

where the $\cos 2 \phi_{\vec{k}}$ factor represents the $d_{x^{2}-y^{2}}$ symmetry of the nematic orders of $n_{c}$ and $n_{s p}$.

We are ready to study the zero-temperature phase diagram with the Zeeman energy term as:

$$
H_{e x t}=-h \sum_{a=x z, y z} \sigma n_{a, \sigma}(i) .
$$

$\mu$ is tuned to reach the filling of $n=3.48$ at $h=0$ in the bilayer bonding bands of $d_{x z}$ and $d_{y z}$ so that the FS 


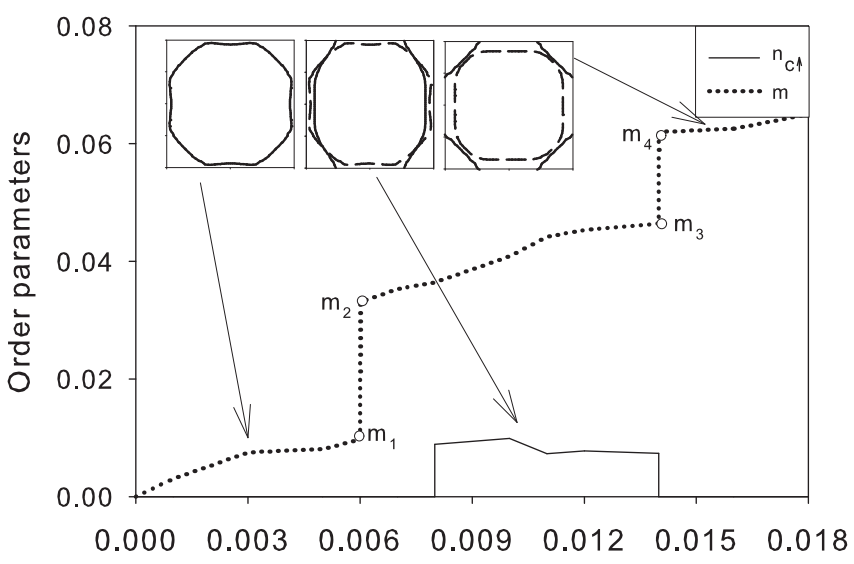

$\mathrm{h}$

FIG. 3: $m$ and the nematic order parameter for majority spin band $\left(n_{c \uparrow}=\left(n_{c}+n_{s p}\right) / 2\right)$ versus the external field $h$. The topologies of the FS of the $\gamma_{+}$band in each phases are sketched in the insets, where the solid and dashed lines represent the FS of the majority and the minority spin bands.

of the $\gamma_{+}$band is very close to the van Hove singularity while that of the $\gamma_{-}$band is far away from the singularity. The interaction parameters are chosen as $(U, V, J, \Delta)=$ $(2.7,2.5,0,0)$, which remarkably reproduce the isotropicnematic-isotropic phase transition as a function of $h$ as we expect from the Ginzburg-Landau analysis.

The magnetization $m$ and the nematic order of the majority spin Fermi surface $n_{c, \uparrow}=\frac{1}{2}\left(n_{c}+n_{s p}\right)$ are depicted in Fig. 3, while that of the minority spin Fermi surface is much smaller (not shown) because its Fermi surface is pushed from the van Hove singularity. As explained in Ref:,$\underline{4}$, the first jump of $m$ distorts the Fermi surface to touch the van Hove singularity points along one of the $a b$ axes, and the second jump restores the four fold symmetry of Fermi surface to cover the singularity in both directions. Compared to Ref. $\underline{\underline{4}}$, the range of $h$ for the nematic state is significantly reduced in agreement with experiment due to inclusion of the magnetic order $m$ selfconsistently in the solution. Furthermore, the first jump of $m$ is larger than the second one as consistent with the experiments. This feature can be understood by the asymmetric profile of the DOS at the van Hove singularity which drops faster in the higher energy side as shown in Fig. 2 After the first transition, part of the FS of the majority spin has moved beyond the van Hove singularity. This reduces the DOS at the second jump and leads to a weaker second jump where the nematic order disappears.

We learned another independent and beautiful work by Raghu et al $\stackrel{45}{\underline{n}}$ which was posted at the same time as ours. The same mechanism is proposed for the nematic state observed in $\mathrm{Sr}_{2} \mathrm{Ru}_{3} \mathrm{O}_{7}$ based on the quasi-one dimensional bands. They used a more detailed band structure and further considered the spin-orbit coupling effect. We further performed the Ginzburg-Landau analysis for the competition between ferromagnetization and nematic ordering.

\section{CONCLUSION AND OUTLOOK}

In this article, we have studied the Fermi liquid instability of the Pomeranchuk type in orbital band systems. Orbital band hybridization significantly enhances the Landau interaction functions in high partial wave channels, thus providing a mechanism for the nematic states or unconventional magnetism from conventional interactions. Consequentially, metamagnetism (ferromagnetism) induces the nematic behavior even with the onsite multi-band Hubbard interactions.

This mechanism is applied to the $t_{2 g}$ system of $\mathrm{Sr}_{2} \mathrm{Ru}_{3} \mathrm{O}_{7}$ by attributing the observed nematic behavior to the hybridized quasi-one dimensional bands of $d_{x z}$ and $d_{y z}$, which is the major difference between our work and Ref $\frac{4,42,43}{}$. Many open questions still need future exploration. In particular, the quick suppression of the nematic behavior with the in-plane magnetic field $h_{\|}$might result from the orbital effect due to the bilayer splitting as pointed out in Ref $\frac{42}{2}$, or from the spin-orbit coupling effect.

The mechanism presented in this article also very general. It is essentially a Berry phase effect which naturally arises from electron systems with non-trivial band structures, such as spin-orbit coupling system and graphene. It can be understood as a conventional interaction acquires a non-trivial nature after projected onto a nontrivial band structure. We further predict that the nematic ordering arises at the ferromagnetic transition in spin-orbit coupling systems as a result of the hybridization between two spin components in the band structure. For example, the in-plane ferromagnetic ordering in the quasi-2D Rashba systems and the surface states of the topological insulators induces the $p$-wave distortion of Fermi surfaces, which will be presented in a later publication ${ }^{46}$.

\section{ACKNOWLEDGEMENT}

We thank X. Dai, J. Hirsch, Y. B. Kim and H. Y. Kee for helpful discussions. C. W. is grateful for E. Fradkin, S. A. Kivelson, and S. C. Zhang for their education on Pomeranchuk instability and encouragement on this project. This work is supported by AROW911NF0810291 and Sloan Research Foundation. 
1 I. I. Pomeranchuk, Soviet Physics JETP 8, 361 (1959).

${ }^{2}$ V. Oganesyan, S. A. Kivelson, and E. Fradkin, Phys. Rev. B 64, 195109 (2001).

3 S. A. Kivelson, I. P. Bindloss, E. Fradkin, V. Oganesyan, J. M. Tranquada, A. Kapitulnik, C. Howald, Rev. Mod. Phys. 75, 1201 (2003).

${ }^{4}$ H. Y. Kee and Y. B. Kim, Phys. Rev. B 71, 184402 (2005).

${ }^{5}$ C. M. Varma, Philos. Mag. 85, 1657 (2005).

6 D. G. Barci and L. E. Oxman, Phys. Rev. B 67, 205108 (2003);

7 D. G. Barci, M. Trobo, V. Fernandez, and L. E. Oxman, Phys. Rev. B 78, 035114 (2008).

8 C. J. Halboth and W. Metzner, Phys. Rev. Lett. 85, 5162 (2000).

9 H. Y. Kee, Phys. Rev. B 67, 73105 (2003).

10 M. J. Lawler, D. G. Barci, V. Fernandez, E. Fradkin, and L. E. Oxman, Phys. Rev. B 73, 085101 (2006).

11 J. Nilsson and A. H. Casto Neto, Phys. Rev. B 72, 195104 (2005).

12 J. Quintanilla and A. J. Schofield, Phys. Rev. B 74, 115126 (2006).

13 L. Dell'Anna and W. Metzner, Phys. Rev. B 73, 045127 (2006).

14 I. Khavkine, C.-H Chung, V. Oganesyan, and H.-Y. Kee, Phys. Rev. B 70, 155110 (2004).

15 H. Yamase and H. Kohno, J. Phys. Soc. Jpn 69, 332 (2000)

16 H. Yamase and H. Kohno, J. Phys. Soc. Jpn. 70, 2733 (2001).

17 H. Yamase, V. Oganesyan, and W. Metzner, Phys. Rev. B 72, $35114(2005)$

18 H. Yamase, Phys. Rev. B 76, 155117 (2007)

19 C. Honerkamp, Phy. Rev. B 72, 115103 (2005).

20 P. Wolfle and A. Rosch, J. of Low Temp. Phys., 147, 165 (2007)

21 C. A. Lamas, D. C. Cabra, and N. Grandi, Phys. Rev. B 78, 115104 (2008).

22 S. A. Kivelson, E. Fradkin, and V. J. Emery, Nature 393, 550 (1998).

${ }^{23}$ E. Fradkin, S. A. Kivelson, E. Manousakis, and K. Nho, Phy. Rev. Lett. 84, 1982 (2000).

24 E. Fradkin and S. A. Kivelson, Phys. Rev. B 59, 8065 (1999).

25 M. P. Lilly, K. B. Cooper, J. P. Eisenstein, L. N. Pfeiffer, and K. W. West, Phy. Rev. Lett. 82, 394 (1999).

${ }^{26}$ K. B. Cooper, M. P. Lilly, J. P. Eisenstein, L. N. Pfeiffer, and K. W. West, Phys. Rev. B 65, 241313 (2002).

27 R. R. Du, D.C. Tsui, H.L. Stormer, L.N. Pfeiffer, K.W.
Baldwin, and K.W. West, Sol. Stat. Comm. 109, 389 (1999).

28 C. Wu and S.C. Zhang, Phys. Rev. Lett. 93, 36403 (2004).

29 C. Wu, K. Sun, E. Fradkin, and S.C. Zhang, Phys. Rev. B 75, 115103 (2007).

30 C. M. Varma and L. Zhu, Phys. Rev. Lett. 96, 036405 (2006).

31 J. E. Hirsch, Phys. Rev. B 41, 6820 (1990).

32 J. E. Hirsch, Phys. Rev. B 41, 6828 (1990).

33 A. V. Chubukov and D. L. Maslov, arXiv.org:0908.4433 (unpublished).

34 S. A. Grigera, R. S. Perry, A. J. Schofield, M. Chiao, S. R. Julian, G. G. Lonzarich, S. I. Ikeda, Y. Maeno, A. J. Mills, and A. P. Mackenzie, Science 294, 329 (2001).

35 S. A. Grigera, P. Gegenwart, R. A. Borzi, F. Weickert, A. J. Schofield, R. S. Perry, T. Tayama, T. Sakakibara, Y. Maeno, A. G. Green, A. P. Mackenzie, Science 306, 1154 (2004).

36 R. A. Borzi, S. A. Grigera, J. Ferrell, R. S. Perry, S. J. S. Lister, S. L. Lee, D. A. Tenant, Y. Maeno, and A. P. Mackenzie, Science 315, 214 (2007).

37 A. M. Berridge, A. G. Green, S. A. Grigera, and B. D. Simons, Phys. Rev. Lett. 102, 136404 (2009).

38 A. J. Millis, A. J. Schofield, G. G. Lonzarich, and S. A. Grigera, Phys. Rev. Lett. 88, 217204 (2002).

39 A. G. Green, S. A. Grigera, R. A. Borzi, A. P. Mackenzie, R. S. Perry, and B. D. Simons, Phys. Rev. Lett. 95, 086402 (2005).

40 A. Tamai, M. P. Allan, J. F. Mercure, W. Meevasana, R. Dunkel, D. H. Lu, R. S. Perry, A. P. Mackenzie, D. J. Singh, Z. X. Shen, and F. Baumberger, Phys. Rev. Lett. 101, 26407 (2008).

${ }^{41}$ K. Iwaya, S. Satow, T. Hanaguri, N. Shannon, Y. Yoshida, S. I. Ikeda, J. P. He, Y. Kaneko, Y. Tokura, T. Yamada, and H. Takagi, Phys. Rev. Lett. 99, 057208 (2007).

42 C. Puetter, H. Doh, and H. Y. Kee, Phys. Rev. B 76, 235112 (2007).

${ }^{43}$ H. Yamase and A. A. Katanin, J.PHYS.SOC.JPN. 76, 073706 (2007).

44 H. v Löhneysen, A. Rosch, M. Vojta, and P. Wölfle, Rev. Mod. Phys. 79, 1015 (2007).

45 S. Raghu, A. Paramekanti, E.-A. Kim, R. A. Borzi, S. A. Grigera, A. P. Mackenzie, and S. A. Kivelson, Phys. Rev. B 79, 214402 (2009).

${ }^{46}$ W. C. Lee and C. Wu, unpublished. 